\title{
La distribución de competencias entre el Estado y las Comunidades Autónomas en materia de asociaciones y de cooperativas ${ }^{1}$
}

\author{
Manuel Carrasco Durán \\ Profesor Titular de Derecho Constitucional \\ de la Universidad de Sevilla
}

\begin{abstract}
SUMARIO: I. EL REPARTO DE COMPETENCIAS EN MATERIA DE ASOCIACIONES: DEBATE DOCTRINAL Y CONCRECIÓN JURISPRUDENCIAL Y NORMATIVA. II. LA JURISPRUDENCIA SOBRE ACTUACIONES CONCRETAS RELATIVAS A LAS ASOCIACIONES, SOBRE ASOCIACIONES ESPECÍFICAS Y SOBRE COOPERATIVAS. III. REFLEXIONES FINALES: EL REPARTO DE COMPETENCIAS EN MATERIA DE ASOCIACIONES PASADO POR EL TAMIZ DE LA REALIDAD
\end{abstract}

Examinar el reparto de competencias en materia de asociaciones resulta de interés por dos motivos: en primer lugar, porque son varias las Comunidades Autónomas donde se están elaborando en este momento proyectos de ley reguladores de las asociaciones en su ámbito territorial, sobre la base del contenido de la Ley Orgánica 1/2002, reguladora del Derecho de Asociación, como es el caso de Andalucía, y, en segundo lugar, porque se trata de una materia que nos sirve de ejemplo práctico para examinar la manera como inciden las competencias del Estado de contenido genérico o transversal, especialmente en esta materia la relativa a la regulación de las condiciones básicas que garanticen la igualdad de todos los españoles en el ejercicio de los derechos y en el cumplimiento de los deberes constitucionales (art. 149.1.1 CE), sobre las competencias de las Comunidades Autónomas, tema este que ha suscitado numerosas polémicas doctrinales y políticas.

En el presente artículo comenzaremos examinando en general el reparto de las competencias relativas a las asociaciones entre el Estado y las Comuni-

\footnotetext{
${ }^{1}$ Este artículo toma su base del texto que presenté como ponencia al Seminario sobre el Derecho de Asociación organizado por el Instituto Andaluz de Administración Pública en Sevilla en el mes de noviembre de 2004. Al texto original de dicha ponencia he añadido otros datos y comentarios que completan las reflexiones que, de forma más sintética, expuse con ocasión de dicho Seminario.
} 
dades Autónomas, para lo cual aludiremos a las principales opiniones doctrinales que se han ocupado de la materia y a la jurisprudencia del Tribunal Constitucional que ha concretado la articulación de las competencias de ambos tipos de ente sobre dicha materia. Asimismo, se examinará brevemente cuál es el estado actual de la normativa estatal y autonómica en materia de asociaciones. Posteriormente, se aludirá a la jurisprudencia del Tribunal Constitucional que ha tratado sobre cuál es el ente titular de la competencia para llevar a cabo determinadas actuaciones, tanto legislativas como ejecutivas, sobre algunos tipos concretos de asociaciones. Al hilo del tratamiento de los temas anteriores, se incluirán también referencias al régimen competencial de las cooperativas. Finalmente, se incluye un capítulo en el que se detallarán unas breves conclusiones en torno a los temas tratados previamente.

\section{EL REPARTO DE COMPETENCIAS EN MATERIA DE ASOCIA- CIONES: DEBATE DOCTRINAL Y CONCRECIÓN JURISPRU- DENCIAL Y NORMATIVA}

Cuando se examinan las normas sobre reparto de competencias entre el Estado y las Comunidades Autónomas en materia de asociaciones destacan dos notas:

a) En primer lugar, ni el Estado ni las Comunidades Autónomas tienen atribuida por la Constitución o por los Estatutos de Autonomía, respectivamente, una competencia genérica sobre asociaciones.

b) Existe, a pesar de todo, una gran dispersión en el tratamiento competencial de las asociaciones. La Constitución y los Estatutos de Autonomía hacen menciones a distintas asociaciones concretas. Así, la Constitución, en sus artículos 6 y 7 , alude a algunas asociaciones específicas, los partidos políticos, los sindicatos y las asociaciones empresariales, significadas por el papel institucional que desempeñan en el funcionamiento del sistema político y de las relaciones laborales. Estos artículos señalan algunos principios que el Estado debe respetar en cuanto al concepto de partidos políticos, sindicatos y asociaciones empresariales que recojan las leyes reguladoras de estas asociaciones y en cuanto a su creación y el ejercicio de su actividad, que deben ser libres dentro del respeto a la Constitución y a la ley, y a su estructura interna y funcionamiento, que deberán ser democráticos. Estas asociaciones, en cuanto que constituyen manifes- 
taciones destacadas del derecho de asociación y, en el caso de los partidos y de los sindicatos, cauce indispensable hoy en día para que sea posible el ejercicio del derecho de participación política (art. $23 \mathrm{CE}$ ) y de la libertad sindical (art. $28 \mathrm{CE}$ ), deben ser reguladas por el Estado, en principio, dentro de los límites que tiene toda regulación de los derechos fundamentales mediante ley orgánica, que debe ceñirse al desarrollo del contenido de los derechos estrictamente derivado de la Constitución. Pero, además, las Comunidades Autónomas no han asumido competencias para la regulación de partidos y sindicatos, lo que convierte en exclusiva la competencia del Estado para regularlas por la vía de la cláusula residual del artículo 149.3 in fine de la Constitución.

También se refiere la Constitución en sus artículos 51 y 52 a las organizaciones de consumidores y usuarios y a las organizaciones profesionales, si bien en términos que no precisan el ente competente para regularlas y disciplinarlas. Ello permite, en particular, que las Comunidades Autónomas ejerzan competencias en relación con la regulación de las asociaciones de defensa de los consumidores y usuarios a partir de sus competencias sobre esta materia, que sus Estatutos de Autonomía les reconocen como exclusiva, de acuerdo con las bases y la ordenación de la actividad económica general y la política monetaria del Estado, en los términos de lo dispuesto en los artículos 38, 131, 149.1.11 y 149.1.13, y sin perjuicio de la política general de precios y de la legislación sobre defensa de la competencia [arts. 12.1.5) del Estatuto de Autonomía de Cataluña; en el mismo sentido se pronuncian los artículos correspondientes de los demás Estatutos de Autonomíaª].

Los Estatutos de Autonomía sí contienen reglas competenciales relativas a asociaciones, si bien pueden dividirse, a este respecto, en dos

\footnotetext{
${ }^{2}$ Vid., solamente a título indicativo, los artículos 30.1.4 del Estatuto de Autonomía de Galicia y 18.1.6 del Estatuto de Autonomía de Andalucía. Solamente el artículo 10.28 del Estatuto de Autonomía del País Vasco introduce una peculiaridad de redacción, ya que reconoce al País Vasco competencia exclusiva sobre defensa del consumidor y del usuario «en los términos del apartado anterior», que se refiere, como condicionantes al ejercicio de la competencia autonómica sobre comercio interior, a la política general de precios, la libre circulación de bienes en el territorio del Estado y la legislación sobre defensa de la competencia. El artículo 22.1.2 del Estatuto de Autonomía de Ceuta y del Estatuto de Autonomía de Melilla solamente reconoce a estas Ciudades Autónomas competencias para la ejecución de la legislación del Estado en materia de defensa de consumidores y usuarios.
} 
grupos. Uno, el formado actualmente por los Estatutos de Autonomía de País Vasco, Cataluña, Andalucía, Comunidad Valenciana y Canarias, que reconocen a estas Comunidades Autónomas competencias, calificadas como exclusivas, sobre fundaciones y asociaciones de carácter docente, cultural, artístico, benéfico-asistencial y similares, que desarrollen principalmente sus funciones en la respectiva Comunidad Autónoma ${ }^{3}$. En el segundo grupo se hallan los Estatutos de Autonomía de las demás Comunidades Autónomas, que aquéllas atribuyen solamente la función ejecutiva, en los términos que establezcan las leyes y normas reglamentarias del Estado, en relación con las asociaciones que desarrollan principalmente sus funciones en la Comunidad Autónoma. Estos Estatutos de Autonomía, así pues, limitan las competencias de las Comunidades Autónomas a las que se refieren a la función ejecutiva y no les atribuyen funciones legislativas, si bien, por otra parte, extienden el objeto material de tales competencias a todas las asociaciones que desarrollen principalmente sus funciones en el ámbito territorial de la Comunidad Autónoma, sin señalar específicamente una lista concreta de asociaciones, como ocurría en los títulos competenciales de las Comunidades Autónomas del primer grupo ${ }^{4}$. Excepciones a este modelo son el Estatuto de Autonomía de Aragón, que reconoce a esta Comunidad Autónoma competencia ejecutiva en relación con la lista de asociaciones característica de las reglas competenciales de los Estatutos de Autonomía del primer grupo, y el Estatuto de Autonomía de Galicia, que no reconoce a esta Comunidad Autónoma competencia alguna sobre la materia de asociaciones, lo que constituye una clara anomalía ${ }^{5}$.

\footnotetext{
${ }^{3}$ Vid. los artículos 10.13 del Estatuto de Autonomía del País Vasco, 9.24 del Estatuto de Autonomía de Cataluña, 13.25 del Estatuto de Autonomía de Andalucía, 31.23 del Estatuto de Autonomía de la Comunidad Valenciana y 30.7 del Estatuto de Autonomía de Canarias tras la reforma llevada a cabo en el mismo por medio de la Ley Orgánica 4/1996, de 30 de diciembre.

${ }^{4}$ Artículos 33.2 del Estatuto de Autonomía de Castilla-La Mancha, 12.1.1 del Estatuto de Autonomía de Murcia, 28.1.4 del Estatuto de Autonomía de Madrid, 26.2 del Estatuto de Autonomía de Cantabria, 12.2 del Estatuto de Autonomía de Asturias, 11.1.13 del Estatuto de Autonomía de La Rioja, 36.1 del Estatuto de Autonomía de Castilla y León, 12.5 del Estatuto de Autonomía de las Islas Baleares y 9.2 del Estatuto de Autonomía de Extremadura.

${ }^{5}$ Actual artículo 39.1.1 del Estatuto de Autonomía de Aragón. Tampoco los Estatutos de Autonomía de las Ciudades Autónomas de Ceuta y Melilla atribuyen a éstas competencias sobre asociaciones.
} 
Además, y como fenómenos distintos al asociativo, pero en cierta medida relacionados con él, los Estatutos de Autonomía atribuyen a las Comunidades Autónomas, de manera uniforme esta vez, competencias exclusivas sobre cooperativas, pósitos y mutuas no integradas en el sistema de la Seguridad Social, con la salvedad de la necesidad de respetar la legislación mercantil ${ }^{6}$. Junto a ello, habría que añadir lo que ya ha sido señalado respecto a la competencia para la regulación de las asociaciones de defensa de los consumidores y usuarios.

Estamos, así pues, ante una materia, la de asociaciones, en la que, a pesar de los procesos de reforma de los Estatutos de Autonomía que accedieron originalmente a la autonomía a través de las vías abiertas por los artículos 143 y 144 de la Constitución, no se ha producido la homogeneidad competencial entre todas las Comunidades Autónomas. De hecho, como puede deducirse fácilmente, el grueso de la polémica acerca de la distribución de competencias en materia de asociaciones se ha producido a la hora de determinar la extensión de las facultades que corresponderían al Estado acerca de la regulación de las asociaciones en relación con las Comunidades Autónomas cuyos Estatutos de Autonomía les atribuyen competencias exclusivas sobre una larga lista de asociaciones.

Ante la falta de una regla que atribuyera en la Constitución al Estado alguna competencia en materia de asociaciones, el debate doctrinal que se produjo en los primeros años de vigencia de la Constitución y de los Estatutos de Autonomía se puede calificar con la cinematográfica expresión de «a la búsqueda de la competencia perdida». En efecto, la doctrina se dedicó a investigar cuál sería el artículo de la Constitución del que pudiera deducirse una competencia a favor del Estado para regular las asociaciones, cuya existencia

\footnotetext{
${ }^{6}$ Artículos 10.23 del Estatuto de Autonomía del País Vasco, 9.21 del Estatuto de Autonomía de Cataluña, 13.20 del Estatuto de Autonomía de Andalucía, 31.21 del Estatuto de Autonomía de la Comunidad Valenciana, 30.24 del Estatuto de Autonomía de Canarias, 35.1.23 del Estatuto de Autonomía de Aragón, 31.1.22 del Estatuto de Autonomía de Castilla-La Mancha, 10.1.23 del Estatuto de Autonomía de Murcia, 26.1.14 del Estatuto de Autonomía de Madrid, 24.26 del Estatuto de Autonomía de Cantabria, 10.1.27 del Estatuto de Autonomía de Asturias, 8.1.12 del Estatuto de Autonomía de La Rioja, 32.1.24 del Estatuto de Autonomía de Castilla y León y 10.26 del Estatuto de Autonomía de las Islas Baleares y 7.1.23 del Estatuto de Autonomía de Extremadura. Hay pequeñas diferencias de redacción en las reglas competenciales correspondientes de algunos de estos Estatutos de Autonomía que en nada modifican la uniformidad de la atribución competencial a todas las Comunidades Autónomas en materia de cooperativas, pósitos y mutualidades.
} 
se daba por supuesta. Tres artículos se trajeron a colación, principalmente, para apoyar esta búsqueda: el 81.1, el 149.1.8 y el 149.1.17.

Argumentaron algunos autores que la atribución a la ley orgánica del desarrollo de los derechos fundamentales y las libertades públicas en el artículo 81.1 de la Constitución podía considerarse título competencial suficiente a favor del Estado para legitimar a este para desarrollar el derecho de asociación. Desde esta perspectiva, habría correspondido al Estado la regulación del derecho de asociación, mientras que las competencias de las Comunidades Autónomas habrían quedado reducidas a funciones ejecutivas de la legislación del Estado, tales como policía y sanción, y, todo lo más, a la normación de aspectos organizativos o que pudieran hacer referencia al favorecimiento, impulso o fortalecimiento del hecho asociativo, como la actividad de fomento de las asociaciones ${ }^{8}$. Ciertamente, la jurisprudencia constitucional ha descartado que el artículo 81.1 de la Constitución sea considerado como norma de atribución competencial a favor del Estado, pero, en la práctica, el Tribunal Constitucional procede a derivar de forma directa de dicho artículo las facultades del Estado para regular los elementos nucleares del contenido de los derechos fundamentales ${ }^{9}$, si bien también es cierto que tales facultades, en la jurisprudencia del Tribunal Constitucional, no tienen la extensión que pretendieron atribuirles quienes defendieron la utilización de este artículo como base competencial para el reconocimiento de competencias al Estado en materia de asociaciones. Nos referiremos con más detalle a este tema al hilo del estudio de la STC 173/1998, que incluimos más adelante.

De igual modo, se argumentó que el título sobre legislación civil del artículo 149.1.8 de la Constitución sería suficiente para atribuir al Estado competencias sobre un buen número de aspectos relativos a las asociaciones, con la

${ }^{7}$ MARÍN LÓPEZ, J. J.; «Comentario al artículo 20», en R. Bercovitz y J. Salas (dirs.); Comentarios a la Ley para la defensa de los Consumidores y Usuarios, Civitas, Madrid, 1992, págs. 510 y ss., y «Sobre la Ley vasca de Asociaciones», Revista Jurídica de Castilla-La Mancha, 16 (1992), 121 y ss.

${ }^{8}$ LÓPEZ-NIETO Y MALLO, F.; La ordenación legal de las asociaciones, Dykinson, Madrid, $4^{\mathrm{a}}$ ed., 2004, pág. 164, y, en términos similares, FERNÁNDEZ FARRERES, G.; Asociaciones y Constitución, Civitas, Madrid, 1987, págs. 141 y 143, y MARTÍN MERCHÁN, D.; «El derecho de asociación y las Comunidades Autónomas», Documentación Administrativa, 195 (1982), 219.

${ }^{9} \mathrm{Vid}$., sobre el efecto que esto provoca en las competencias autonómicas, JIMÉNEZ ASENSIO, R.; La ley autonómica en el sistema constitucional de fuentes del derecho, Marcial Pons, Institut d'Estudis Autonómics, IVAP, Madrid, 2001, págs. 163 y ss. 
nota adicional de que la competencia del Estado sobre legislación civil tiene carácter de exclusiva ${ }^{10}$. No obstante, el Tribunal Constitucional, en sus sentencias 218/1988 y 5/1996, redujo drásticamente el alcance que algunos autores habían advertido en el título sobre legislación civil, al establecer límites al contenido y la eficacia del pacto asociativo y revisar desde una perspectiva restrictiva la teoría del contrato civil.

Quedó, de esta forma, el título del artículo 149.1.1 de la Constitución sobre la regulación de las condiciones básicas que garanticen la igualdad de todos los españoles en el ejercicio de los derechos y en el cumplimiento de los deberes constitucionales como aquel en el que habría de fundarse primordialmente la competencia del Estado para regular las asociaciones. Además, colateralmente, hay que destacar la incidencia en el régimen de las asociaciones de otros títulos competenciales del Estado, tales como el relativo a la competencia exclusiva sobre legislación mercantil (art. 149.1.6 CE), de importancia para determinar el régimen competencial aplicable a la actividad de asociaciones cuya actuación se desarrolle en el campo mercantil, o el relativo a la competencia exclusiva sobre legislación procesal (art. 149.1.6 CE), a través del cual el Estado puede determinar, por ejemplo, la legitimación activa de las asociaciones para intervenir en los procesos.

Por otra parte, otros autores, entre los que destaca Pablo Lucas Murillo, desde una perspectiva contraria, consideraron que los títulos competenciales de carácter exclusivo sobre diversas asociaciones que algunos Estatutos de $\mathrm{Au}-$ tonomía reconocen a determinadas Comunidades Autónomas eran suficientes para deducir a favor de estas una competencia para la regulación genérica de las asociaciones. El argumento principal a favor de esta postura era que estos títulos recogían sustancialmente todas las asociaciones a las que se refería la Ley 191/1964, de 24 de diciembre, Reguladora de las Asociaciones, vigente hasta el año 2002, y dejaban fuera solamente asociaciones tales como los partidos políticos, los sindicatos y las asociaciones empresariales, que tampoco la Ley de Asociaciones regulaba ${ }^{11}$.

Como suele ocurrir, ha tenido que ser también en esta materia el Tribunal Constitucional el que ordenara esta acumulación de referencias desorde-

\footnotetext{
${ }^{10}$ MARÍN LÓPEZ, J. J.; «Comentario al artículo 20», ob. cit., pág. 513.

${ }^{11}$ MURILlO DE LA CUEVA, P. L.; El derecho de asociación, Tecnos, Madrid, 1996, págs. 275 y ss.
} 
nadas a las asociaciones y de interpretaciones dispares sobre su régimen competencial. Y lo ha hecho, especialmente, en la sentencia 173/1998, sobre la Ley de Asociaciones del País Vasco:

La sentencia argumenta que las facultades del Estado para llevar a cabo la regulación de las asociaciones provienen principalmente de dos normas:

a) Ante todo, el artículo 81.1 de la Constitución, pero sobre la base de que este no atribuye al Estado título competencial alguno. Solamente lo faculta para desarrollar normativamente los aspectos esenciales para la definición del derecho de asociación, en abstracto, expresado en el artículo 22 de la Constitución, esto es, la previsión de su ámbito y la fijación de sus límites en relación con otras libertades constitucionalmente protegidas. Desde este soporte, la regulación estatal sólo puede desarrollar la libertad de creación de asociaciones y de adscripción a las ya creadas, la libertad de no asociarse y de dejar de pertenecer a las asociaciones, la libertad de organización y funcionamiento interno y los derechos de los asociados frente a las asociaciones o de los participantes que quieran incorporarse. En otras palabras, el artículo 81.1 de la Constitución permite al Estado referirse solamente a la titularidad del derecho, las facultades elementales que lo integran, el alcance del ejercicio del derecho en las relaciones inter privatos y las garantías fundamentales y límites del derecho en relación con otros derechos y libertades, aspecto en el que se engloba la mención de las asociaciones constitucionalmente proscritas, de las restricciones al derecho de asociación de determinadas personas y de los límites a la libertad de no asociarse.

b) El artículo 149.1.1 de la Constitución permite al Estado regular las posiciones jurídicas fundamentales (facultades elementales, límites esenciales, deberes fundamentales, prestaciones básicas, premisas o presupuestos previos) de cara al ejercicio del derecho de asociación. La sentencia es interesante porque diferencia nítidamente el distinto alcance de las facultades que el Estado puede ejercer desde las bases normativas integradas por los artículos 81.1 y 149.1.1 de la Constitución. Así, el último de dichos artículos amplía el campo de actuación del Estado en materia de asociaciones, al permitirle definir el concepto legal de asociación y su régimen jurídico externo, lo que atañe al nacimiento de la personalidad jurídica, la capacidad jurídica y de obrar, el régimen de responsabilidad y las causas y efectos de la disolución de la asociación, si bien la regulación estatal debe tener 
solamente el contenido mínimo necesario para garantizar una igualdad básica en el ejercicio del derecho de asociación en todo el Estado, dejando la concreción de dicha normativa y su ejecución a las Comunidades Autónomas.

d) Facultades adicionales le vienen atribuidas al Estado en relación con los aspectos civiles, administrativos, procesales, fiscales y penales que inciden en el régimen de las asociaciones, en virtud de las competencias específicas de aquel sobre estos ámbitos materiales.

En relación con las competencias de las Comunidades Autónomas, la sentencia establece tres principios importantes:

a) Del título sobre asociaciones de los Estatutos de Autonomía no es posible deducir una competencia genérica de aquellas sobre las asociaciones. La competencia de las Comunidades Autónomas alcanza solamente a aquellas específicas asociaciones mencionadas en las reglas competenciales que, al respecto, recogen los Estatutos de Autonomía.

b) El contenido de las competencias de las Comunidades Autónomas debe ser interpretado de conformidad con la calificación de exclusivas que los Estatutos de Autonomía dan a sus facultades sobre las asociaciones. De esta forma, las competencias autonómicas sobre las asociaciones alcanzan también facultades legislativas.

c) A pesar de todo, el ejercicio de las competencias exclusivas de las Comunidades Autónomas sobre las asociaciones debe respetar el marco regulador genérico que establezca el Estado mediante el ejercicio de las facultades que le atribuyen los artículos 81.1 y 149.1.1 de la Constitución.

Sobre estas bases, la sentencia examina de forma particularizada los artículos de la Ley de Asociaciones del País Vasco que habían sido impugnados en el recurso. Interesa detenerse en la respuesta que la sentencia da a cada impugnación, ya que de ellas pueden extraerse interesantes conclusiones respecto a la técnica legislativa utilizable por las leyes de asociaciones de las Comunidades Autónomas:

a) La Ley de Asociaciones de la Comunidad Autónoma puede concretar su contenido en un tono genérico, como si estuviera regulando 
las asociaciones de modo general, ya que basta que en su primer artículo precise que la Ley se aplica a las asociaciones que caen bajo el manto del título competencial de aquella para entender que la Ley se aplica sólo a ellas. De hecho, este es el tono que emplean las leyes autonómicas de asociaciones existentes en la actualidad, que son la Ley de Asociaciones del País Vasco de 12 de febrero de 1988, la Ley de Asociaciones de Cataluña de 1 de julio de 1997 y la Ley de Asociaciones de Canarias de 10 de marzo de 2003. Con el estilo generalista indicado, estas leyes regulan los principios fundamentales del régimen asociativo (objeto, naturaleza, principios, régimen jurídico, constitución, estatutos, denominación, domicilio social, duración, unión y responsabilidad frente a terceros de las asociaciones), el régimen jurídico de los asociados (derechos, deberes, infracciones, etc.), la organización, disolución, liquidación, inscripción, relaciones con la Administración, declaración de interés público, medidas de apoyo y obligaciones documentales de las asociaciones, y el órgano autonómico de participación y consulta de la Administración de la Comunidad Autónoma para el ejercicio de sus competencias en la materia, entre otros aspectos.

b) La Ley de Asociaciones de la Comunidad Autónoma puede contener normas cuyo objetivo sea enunciar el contenido del derecho de asociación en coherencia con lo reconocido en el artículo 22 de la Constitución y sin añadir a ese derecho ningún contenido normativo propio. De igual manera, puede contener normas que sean la aplicación al derecho de asociación de otros derechos constitucionales, como aquella según la cual la condición de miembro de una determinada asociación no puede suponer motivo de discriminación, favor o ventaja por parte de los poderes públicos.

c) Sin embargo, la Ley de Asociaciones de la Comunidad Autónoma se excede de su contenido posible cuando prevé que la organización y funcionamiento de las asociaciones sean democráticas o que la constitución de asociaciones se lleve a cabo con respeto al pluralismo y los principios democráticos. Estima el Tribunal Constitucional que la Constitución no impone que las asociaciones deban observar estas notas en su organización, funcionamiento y organización, que sólo contempla aquella en sus artículos 6 y 7 respecto de los partidos políticos, sindicatos y asociaciones empresariales, y que, al tratarse de condiciones genéricas de constitución y reconocimiento legal de una asociación y de un límite a la constitución de asociaciones, estas de- 
terminaciones corresponden al Estado, ya que entran en el ámbito de la reserva de la Ley Orgánica del artículo 81 de la Ley Orgánica ${ }^{12}$.

d) Es posible establecer que las asociaciones creadas en el ámbito autonómico se rigen por lo dispuesto en la ley autonómica y el reglamento autonómico que la desarrolle, en cuanto se entiende que excluir la ley del Estado de esta mención no altera el sistema de fuentes y que las normas del Estado, por lo tanto, mantienen su vigencia, dentro del ámbito de competencia de aquel.

e) La Ley autonómica puede establecer normas sobre el concepto y la constitución de asociaciones y exigir un número mínimo de socios para la constitución de la asociación, siempre que estas determinaciones respeten lo dispuesto al respecto en la Ley estatal.

f) Puede la Ley determinar quiénes pueden ejercer el derecho de asociación, siempre que con ello no limite el ámbito de aquellos que, según la Ley del Estado, pueden ejercerlo.

g) Corresponde al Estado determinar si la constitución de una asociación exige escritura pública. En este aspecto, la norma de la Comunidad Autónoma debe respetar lo dispuesto en la norma estatal. La Comunidad Autónoma puede establecer normas particulares sobre la inscripción de la asociación en el Registro (por ejemplo, pautas relativas al procedimiento de inscripción de las asociaciones en el Registro administrativo correspondiente) y sobre los efectos de dicha inscripción,

\footnotetext{
${ }^{12}$ Con todo, el artículo 2 de la Ley de Asociaciones de Cataluña de 18 de junio de 1997 establece que «la organización interna y el funcionamiento de las asociaciones deben ser democráticos, con pleno respeto al pluralismo». No se trata de un defecto atinente sólo a la previsión del carácter democrático de las asociaciones. Lo cierto es que la Ley de Asociaciones de Cataluña fue aprobada con anterioridad a que el Tribunal Constitucional dictara su STC 173/1998 y su contenido toma como modelo, precisamente, el de la Ley de Asociaciones del País Vasco, en aquel tiempo la única ley integral sobre asociaciones dictada en el ámbito del Estado con posterioridad a la entrada en vigor de la Constitución. Esto puede explicar que la Ley catalana incurra, de hecho, en muchos de los defectos de inconstitucionalidad, por falta de competencia de la Comunidad Autónoma, que el Tribunal Constitucional advirtió en la Ley de Asociaciones del País Vasco mediante la sentencia citada. No obstante, la Ley de Asociaciones de Canarias, a pesar de haber sido aprobada con posterioridad a la STC 173/1998, señala en su artículo 3.2 que, en el ámbito canario, «las asociaciones se regirán en su estructura interna y funcionamiento por los criterios de democracia, pluralismo y respeto a la dignidad de las personas». La exigencia de una organización interna y un funcionamiento democráticos de las asociaciones está recogida hoy en día en el artículo 2.6 de la Ley Orgánica 1/2002, reguladora del Derecho de Asociación.
} 
siempre que sean compatibles con la legislación del Estado, a la que corresponde determinar los efectos de la inscripción en relación con la adquisición o no de la personalidad jurídica por parte de aquellas.

h) Corresponde también a la Comunidad Autónoma establecer y regular su Registro de asociaciones.

i) No puede la Comunidad Autónoma remitir al proceso especial contencioso-administrativo para la protección de los derechos fundamentales el recurso contra los actos de la autoridad encargada de llevar el Registro de asociaciones, ya que esta norma integra el campo de la competencia exclusiva del Estado sobre legislación procesal. Por los mismos motivos, excede la competencia de aquella regular la legitimación activa de las asociaciones, la legitimación para impugnar sus acuerdos, los plazos de las acciones correspondientes, la habilitación judicial de los libros y la atribución de los asuntos relativos a asociaciones a uno u otro orden jurisdiccional. Sin embargo, la STC 15/1989 estima que restringir la legitimación de las asociaciones de consumidores y usuarios para iniciar e intervenir en los procedimientos administrativos para persecución del fraude en materia de oferta, promoción y publicidad falsa o engañosa de productos, actividades o servicios las «constituidas de acuerdo con lo establecido en esta Ley ${ }^{12 \mathrm{bis}}$ » supone una restricción indebida del contenido del derecho de asociación de aquellas que, encontrándose legalmente constituidas de acuerdo con la legislación que le es aplicable, no lo hayan sido en cambio de acuerdo con la Ley general. De esta manera, la legitimación activa en el recurso contencioso-administrativo debe ser reconocida a todas las asociaciones, incluyendo aquellas constituidas de acuerdo con la respectiva ley autonómica.

j) Corresponde al Estado establecer las normas sobre capacidad de las personas de cara a la constitución de las asociaciones, pero la Comunidad Autónoma puede precisar tales normas siempre que lo haga de forma compatible con lo dispuesto en la ley del Estado.

k) Puede también la Comunidad Autónoma regular la disolución de las asociaciones siempre que respete lo dispuesto en la norma del Estado.

12bis La sentencia se refiere a la Ley 26/1984, de 19 de julio, General para la Defensa de los Consumidores y Usuarios. 
1) Corresponde a la Comunidad Autónoma regular el régimen interno de las asociaciones de su competencia, lo que incluye su organización y funcionamiento interno, con respeto al principio de libertad en su organización y funcionamiento, sus Estatutos (contenido, modificaciones e inscripción) y los derechos y deberes mínimos de los socios. Así, por ejemplo, si de la ley autonómica se dedujera el derecho de todos los socios a participar sin distinciones en todos los órganos de gobierno de la asociación, esto haría la norma correspondiente inconstitucional, por contradecir lo previsto respecto a tal extremo en la ley estatal, por las mismas razones expuestas en el apartado c) anterior.

m) Corresponde a la Comunidad Autónoma regular las uniones de asociaciones y establecer la aplicación de su ley de asociaciones a las asociaciones de alumnos y de padres de alumnos.

El marco regulativo fundamental de las asociaciones está conformado actualmente, en el plano estatal, por la Ley Orgánica 1/2002, de 22 de marzo, Reguladora del Derecho de Asociación ${ }^{13}$, que ha derogado la anterior Ley 191/1964, Reguladora de las Asociaciones, y, en el plano autonómico por la Ley de Asociaciones del País Vasco de 12 de febrero de $1988^{14}$, la Ley de Asociaciones de Cataluña de 1 de julio de $1997^{15}$ y la Ley de Asociaciones de Canarias de 10 de marzo de 2003. Son estas tres las únicas Comunidades Autónomas que han regulado de forma genérica las asociaciones ${ }^{16}$.

${ }^{13}$ La Ley 62/2003, de 23 de diciembre, ha modificado el artículo 32.1.a) de la Ley 1/2002. La Ley ha sido desarrollada por el Real Decreto 1497/2003, de 28 de noviembre, relativo al Registro Nacional de Asociaciones y a sus relaciones con los restantes Registros de Asociaciones, y por el Real Decreto 1740/2003, de 19 de diciembre, sobre los procedimientos relativos a las asociaciones de utilidad pública.

${ }^{14}$ La Ley de Asociaciones del País Vasco ha sido afectada por la Ley vasca de 31 de mayo de 1990, reguladora de las Tasas y Precios Públicos, que ha derogado su artículo 10.5. Además, el fallo de la STC 173/1998 declaró inconstitucionales varios artículos de ella.

${ }^{15}$ Esta ley se encuentra recurrida al Tribunal Constitucional mediante el recurso de inconstitucionalidad 3914/1997, promovido por el Presidente del Gobierno. El Tribunal Constitucional aún no se ha pronunciado sobre este recurso. La Ley ha sido afectada por la Ley del Parlamento de Cataluña 11/2005, de 7 de julio, que ha modificado su artículo 24.

${ }^{16}$ En cambio, las fundaciones han merecido mayor atención del legislador autonómico. Regulan las fundaciones, en el ámbito autonómico, la Ley de Galicia 7/1983, de 22 de junio, modificada por la Ley 11/1991, de 8 de noviembre, la Ley del País Vasco 12/1994, de 17 de junio, la Ley de la Comunidad de Madrid 1/1998, de 2 de marzo, la Ley de Canarias 2/1998, de 6 de abril, 
Para terminar el examen de la normativa aplicable con repercusión en la aplicación del reparto de competencias, conviene hacer mención a los Reales Decretos sobre traspaso de funciones y servicios de la Administración del Estado a las Comunidades Autónomas en materia de asociaciones. Como regla general, estos Reales Decretos tienen un contenido muy similar en relación con las distintas Comunidades Autónomas. Puede tomarse como modelo de ellos el Real Decreto 304/1985, de 6 de febrero, relativo a la Comunidad Autónoma de Andalucía, que ha traspasado a dicha Comunidad Autónoma la inscripción de las asociaciones objeto de la competencia de aquella, previa comunicación al Ministerio del Interior, que puede oponer objeciones vinculantes, y ha previsto la remisión al Ministerio del Interior de la documentación para la inscripción de aquellas en el Registro nacional y de las peticiones de declaración de utilidad pública y de integración en organismos de carácter internacional o de adopción de denominaciones alusivas a las mismas, en este último caso junto con un informe-propuesta respecto a lo solicitado. No obstante, y como excepción, el Real Decreto relativo a Cataluña (Real Decreto 3526/1981, de 29 de diciembre) sólo prevé que el Ministerio del Interior emita sugerencias u observaciones pertinentes en relación con la inscripción de la asociación y no precisa a qué asociaciones se aplica, si sólo a las de ámbito autonómico o también a las de ámbito nacional, y el de traspaso al País Vasco (Real Decreto 2590/1985, de 18 de diciembre) no recoge ni la necesidad de enviar al Ministerio del Interior la comunicación antes señalada ni que aquel pueda enviar objeciones u observaciones a la inscripción en relación con las asociaciones que haya que inscribir en el Registro autonómico. Sólo prevé esto para aquellas que haya que inscribir en el Registro nacional.

Ciertamente, la jurisprudencia del Tribunal Constitucional viene considerando, como regla general, que los Reales Decretos sobre traspaso de funciones y servicios de la Administración del Estado a las Administraciones autonómicas no pueden operar como norma encargada de precisar el reparto de competencias entre el Estado y las Comunidades Autónomas. La disparidad en el contenido de los Reales Decretos mencionados relativos al País Vasco, a Cataluña y al resto de las Comunidades Autónomas, sin embargo, apoya la impresión de que aquellos están siendo utilizados, en la práctica, como normas

que sustituye a la Ley 1/1990, de 29 de enero, la Ley de la Comunidad Valenciana 8/1998, de 9 de diciembre, la Ley de Cataluña 5/2001, de 2 de mayo, que sustituye a la Ley 1/1982, de 3 de marzo, la Ley de Castilla y León 13/2002, de 15 de julio, reformada por la Ley 12/2003, de 3 de octubre, y la Ley de Andalucía 10/2005, de 31 de mayo. En el ámbito del Estado, las fundaciones están reguladas por la Ley 50/2002, de 26 de diciembre. A ello hay que añadir las normas reglamentarias sobre organización y funcionamiento de los Registros de Fundaciones, tanto en el ámbito estatal como en el autonómico. No entramos en el examen de esta normativa, ya que rebasa el marco de este trabajo. 
delimitadoras de competencias, fenómeno que, además, no es exclusivo de la materia de asociaciones y que el Tribunal Constitucional, en algunas ocasiones, parece haber aceptado, a pesar de que sus pronunciamientos más genéricos sobre la cuestión vayan, como se ha indicado, por una línea distinta ${ }^{17}$.

\section{LA JURISPRUDENCIA SOBRE ACTUACIONES CONCRETAS RE- LATIVAS A LAS ASOCIACIONES, SOBRE ASOCIACIONES ES- PECÍFICAS Y SOBRE COOPERATIVAS}

Junto a la precisión del reparto general de competencias legislativas sobre asociaciones que acabamos de examinar, el Tribunal Constitucional ha concretado también el régimen competencial de determinadas actuaciones públicas de carácter legislativo o ejecutivo aplicables a las asociaciones y el reparto de competencias sobre cooperativas y sobre algunas asociaciones singularizadas por su objeto social o por algunas características específicas.

a) En cuanto a las subvenciones a las asociaciones, el Tribunal Constitucional tiende a considerar que su régimen jurídico sigue el reparto de competencias establecido en relación con la materia respecto a la cual las asociaciones desarrollan su actuación. El Estado, en principio, puede asignar sus fondos presupuestarios libremente, pero sólo puede detallar la regulación de los elementos fundamentales de las subvenciones en la medida en que se lo permita la competencia que tenga sobre las materias a la que afecte la actuación de las asociaciones, y debe respetar, asimismo, las competencias de las Comunidades Autónomas para regular dichas subvenciones con la amplitud que les permitan los títulos competenciales de los que aquellas sean titulares sobre las mismas materias. Ciertamente, en la mayoría de los casos, pese a tratarse de materias objeto de competencias reconocidas en los Estatutos de Autonomía a las Comunidades Autónomas como exclusivas, el Estado dispondrá de algún título competencial con el que incidir, colateralmente, en la regulación de dichas materias, pero esta incidencia debe estar limitada a las facultades que le permita ejercer dicho título competencial y no deberá alcanzar un grado de detalle tal que llegue a vaciar la competencia autonómica.

\footnotetext{
${ }^{17}$ De acuerdo con la STC 88/1987, FJ 3, «si está claro que dichos Reales Decretos no pueden alterar la distribución de competencias establecida en la Constitución y en los Estatutos de Autonomía, sí son medios idóneos para concretar las formas, modos y procedimiento para el ejercicio de las respectivas competencias estatales y autonómicas.»
} 
Además, en estos casos el Estado deberá territorializar las partidas presupuestarias previstos para las subvenciones.

En particular, la STC 133/1992 trata sobre la regulación de unas subvenciones estatales a asociaciones de consumidores y usuarios. La sentencia señala que, pese a la competencia exclusiva de las Comunidades Autónomas en esta materia de defensa del consumidor y usuario, de acuerdo con las bases y la ordenación de la actuación económica general y la política monetaria del Estado, en los términos de lo dispuesto en los artículos $38,131,149.1 .11$ y 149.1.13, y sin perjuicio de la política general de precios y de la legislación sobre defensa de la competencia (art. 12.1.5 del Estatuto de Autonomía de Cataluña, cuyo Consejo Ejecutivo presentaba el conflicto de competencias resuelto por dicha sentencia), esta materia es pluridisciplinar y nos sitúa también «ante cuestiones propias de la legislación civil y mercantil, de la protección de la salud (sanidad) y seguridad física, de los intereses económicos y del derecho a la información y a la educación en relación con el consumo, de la actividad económica y, en fin, de otra serie de derechos respecto de los cuales pudiera corresponder al Estado la regulación de las condiciones básicas que garanticen la igualdad en su ejercicio y en el cumplimiento de sus deberes (art. 149, en sus núms. 1, 6, 8, 10, 13, 16 y $29 \mathrm{CE}$, principalmente) ${ }^{18}$ ». De esta manera, el Estado, según la sentencia, puede determinar el destino de los fondos que tenga previsto aplicar a las subvenciones a asociaciones de consumidores y usuarios de manera global, por sectores o subsectores enteros de la actividad, y puede también prever la necesidad de que las asociaciones estén inscritas para poder recibir las subvenciones, pero corresponde a las Comunidades Autónomas la regulación de las finalidades específicas a las que deban ir destinadas tales subvenciones, de los criterios de reparto y del procedimiento de otorgamiento de las mismas.

Sin embargo, la STC 102/1995 admite que las facultades del Estado con vistas a detallar la regulación de las subvenciones dirigidas a las asociaciones conservacionistas sin ánimo de lucro para adquisición de terrenos o a titulares de terrenos para programas de conservación son mayores, dado que en medio ambiente el Estado tiene una competencia específica sobre la legislación básica de protección de aquel, que coincide en la misma materia con las que las Comunidades Autónomas tienen para establecer normas adicionales de protección (art. 149.1.23 CE).

${ }^{18}$ La sentencia cita lo señalado al respecto por la STC 15/1989. 
b) En la STC 157/1992 señala el Tribunal Constitucional que el título competencial de las Comunidades Autónomas sobre juventud sólo permite a aquellas establecer medidas de fomento en relación con las asociaciones que se dediquen a temas de juventud, pero no a regular dichas asociaciones. Este pronunciamiento se halla determinado por el hecho de que en el momento en que se dictó el Decreto impugnado, procedente de las Islas Baleares, esta Comunidad Autónoma carecía aún de competencia normativa sobre las asociaciones.

c) La STC 53/1988 señala que el Estado no puede atribuirse, desde su competencia sobre fomento y coordinación general de la investigación científica y técnica (art. 149.1.15 CE) o sobre la coordinación sanitaria (art. 149.1.16 CE), la facultad de pedir datos a las asociaciones científicas de carácter sanitario que se refieran específicamente a su carácter de asociación y no a su actividad investigadora o sanitaria, ya que ello supone una interferencia en el ámbito propio de la autonomía de las Comunidades Autónomas delimitado por sus competencias exclusivas respecto a determinadas asociaciones. La Administración del Estado debe recabar la información de la Administración autonómica y no directamente de los particulares. En este caso, el Tribunal Constitucional tendió a interpretar el título competencial autonómico como atributivo de una competencia genérica sobre las asociaciones, ya que las asociaciones de carácter investigador y sanitario no aparecen mencionadas expresamente en el artículo 10.13 del Estatuto de Autonomía del País Vasco, cuyo Gobierno había presentado el conflicto de competencias resuelto por la sentencia, salvo que se las considere como asociaciones asistenciales o como asociaciones «similares» a las enunciadas en aquel.

d) Las Entidades de gestión de los derechos de propiedad intelectual constituyen organizaciones específicamente diferenciadas en atención al objeto de su actividad y particular régimen jurídico frente a las asociaciones, por lo que no pueden identificarse con las asociaciones a efectos competenciales (STC 196/1997).

Una problemática especial plantean las cooperativas, a las cuales nos referiremos brevemente para contrastar su régimen competencial con el de las asociaciones, aun admitiendo la peculiaridad de este tipo de entes en relación con las asociaciones. La STC 72/1983, sobre la Ley del País Vasco sobre Coope- 
rativas de 11 de febrero de 1982, atribuye a la Comunidad Autónoma la regulación de las actuaciones que lleven a cabo las cooperativas como tales cooperativas, en virtud de sus competencias exclusivas sobre esta materia, si bien deben hacer tal regulación respetando la legislación mercantil en lo que sea aplicable a aquellas. Se refiere este pronunciamiento a las relaciones internas que se establecen entre la cooperativa y sus socios, que dan su carácter específico a aquellas y que son independientes de su actividad comercial, la cual, en el marco de las cooperativas, se vuelca hacia el exterior de ellas y resulta instrumental de las mismas. Así, la competencia de la Comunidad Autónoma sobre cooperativas le permite regular aquellas siempre que su actividad societaria típica se desarrolle en territorio, independientemente de las relaciones que tenga, hacia fuera y en el plano mercantil, con terceros radicados fuera de dicho territorio. La competencia de la Comunidad Autónoma sobre cooperativas permite a aquella precisar las reglas mediante las que debe determinarse el domicilio de las cooperativas o regular la constitución de las cooperativas de forma distinta a lo dispuesto en la legislación estatal sobre esta materia, siempre que la forma de constitución prevista por la Comunidad Autónoma para las cooperativas sea conforme a lo dispuesto en la legislación mercantil. La Comunidad Autónoma, igualmente, puede establecer que la cooperativa se entienda constituida en el momento de su inscripción en el Registro de Cooperativas correspondiente. A este respecto, la legislación autonómica solamente debe respetar los actos que la Ley del Estado considere de obligada inscripción en el Registro Mercantil, ya que la regulación de este atañe a la legislación mercantil, que es competencia del Estado ${ }^{19}$.

En cuanto a las cooperativas de crédito, el régimen competencial tiene una especial dificultad, debido a que en ellas confluyen aspectos crediticios y cooperativos, en los que, a su vez, inciden componentes laborales y mercantiles. En lo que atañe a estos entes, en particular, según señala la STC 155/1993, la competencia exclusiva que en materia de cooperativas tiene la Comunidad

\footnotetext{
${ }^{19}$ En la actualidad, la norma reguladora de las cooperativas a escala estatal es la Ley 27/1999, de 16 de julio, de Cooperativas, si bien la Ley 22/2003, de 9 de julio, Concursal, ha derogado el artículo 73.7 y la disposición adicional cuarta de ella y ha modificado su artículo 41.d), la Ley 44/2002, de 22 de noviembre, ha modificado su artículo 5, el Real Decreto-ley 10/2000, de 6 de octubre, sobre Medidas Urgentes de Apoyo a la Agricultura, Pesca y Transporte ha modificado su artículo 93.4, el Real Decreto Legislativo 5/2000, de 4 de agosto, del Texto Refundido de la Ley sobre Infracciones y Sanciones en el Orden Social ha derogado sus artículos 114 y 115, y la Ley 55/1999, de 29 de diciembre, sobre Medidas Fiscales, Administrativas y del Orden Social, ha modificado su artículo 104. Desarrollan dicha ley el Real Decreto 136/2002, de 1 de febrero, que aprueba el Reglamento del Registro de Sociedades Cooperativas, y el Real Decreto 219/2001, de 2 de marzo, sobre Organización y Funcionamiento del Consejo para el Fomento de la Economía Social.
} 
Autónomas debe conjugarse con la competencia del Estado para fijar las bases de la ordenación del crédito (art. 149.1.11 CE), bases que «deben contener tanto las normas reguladoras de la estructura, organización interna y funciones de los diferentes intermediarios financieros, como aquellas otras que regulan aspectos fundamentales de la actividad de tales intermediarios ...» (STC 1/1982, FJ 3, y reiterando esta afirmación entre otras, SSTC 49/1988, FJ 2, y 135/1992, FJ 1). Las Comunidades Autónomas tienen, sobre este fundamento, competencia para regular el régimen jurídico de las cooperativas de crédito, pero, con arreglo a las singulares características de aquellas, en las que confluyen al menos aspectos crediticios y cooperativos -en los que, a su vez, inciden aspectos laborales y mercantiles (STC 134/1992, FJ 2)-, la normativa autonómica será complementaria de la estatal dictada al amparo del artículo 149.1.11 de la Constitución y de directa aplicación en los aspectos estrictamente cooperativos cuya regulación, por no tener carácter mercantil o laboral (art. 149.1.6 y $7 \mathrm{CE}$ ), corresponda a la competencia exclusiva de las Comunidades Autónomas.

Corresponde, así, al Estado determinar cuándo se debe utilizar la denominación «Cooperativa de Crédito», pues el Tribunal Constitucional entiende que en cuanto a este aspecto tiene preferencia el título sobre ordenación del crédito, más específico que el relativo a cooperativas. De igual forma, corresponde al Estado determinar si las cooperativas de crédito pueden o no concertar operaciones con terceros no socios, al entender la sentencia que esta decisión tiene un carácter íntimamente relacionado con la configuración y definición de dichas entidades, la aprobación de las normas conectadas a la política de solvencia de las cooperativas de crédito y el establecimiento de criterios para la distribución o reparto de los beneficios del ejercicio, que caracterizan la singularidad jurídica de estas cooperativas en relación con otras entidades crediticias. Por el contrario, el Estado no puede determinar la aplicación del excedente a un fondo de educación y promoción que tiene por objeto satisfacer las necesidades de formación y educación de los socios empleados en los principios cooperativos y que, por tanto, entra de lleno en el campo propio de la competencia sobre cooperativas.

La nota más llamativa de esta sentencia es que utiliza la singularidad jurídica de las cooperativas en relación con otras entidades crediticias para atribuir al Estado la regulación de todo aquello que ataña a dicha singularidad,

En el ámbito autonómico, destaca la Ley 2/1999, de 31 de marzo, de Sociedades Cooperativas Andaluzas, modificada en varios de sus artículos por la Ley 3/2002, de 16 de diciembre. 
cuando, precisamente, parece que la regulación de los aspectos en los que consista la singularidad jurídica de las cooperativas debería pertenecer a las Comunidades Autónomas, que tienen la competencia específica sobre aquellas ${ }^{20}$.

Corresponde también al Estado autorizar la constitución de cooperativas de crédito, establecer la necesidad de proceder a la inscripción de la cooperativa y de sus altos cargos en los correspondientes Registros del Banco de España y determinar los órganos sociales de las cooperativas desde la perspectiva de exigencias mínimas que se imponen con el carácter de bases de la ordenación del crédito. Por el contrario, resulta inconstitucional que el Estado determine en detalle la forma de voto de los socios y disponer que el Consejo Rector designará, contratará y destituirá al Director General, ya que el detalle de esta norma excede el ámbito de las bases de la ordenación del crédito.

La STC 275/2000 considera de competencia del Estado la inscripción en el Registro de Cooperativas del Estado cuando se trate de cooperativas de crédito cuyo ámbito de actividad exceda del territorio de una Comunidad Autónoma, la autorización del Banco de España a cambios significativos en las aportaciones societarias, la necesidad de que el Estado autorice la concentración de riesgos cuando sobrepase determinados límites, la inscripción en el registro anterior de los altos cargos y la autorización en determinados supuestos de carácter supracomunitario, mientras que considera inconstitucional la norma que fijaba la composición mínima del Consejo Rector en cinco miembros, dos de los cuales pueden ser no socios, al considerar que esta normativa estatal tiene un exceso de detalle.

Igualmente, según la STC 96/1996, se aplican a las cooperativas de crédito las normas básicas sobre sanciones a las entidades de crédito, lo que no impide su modulación por parte de las leyes autonómicas, siempre que se respeten los límites constitucionales, así como que las Comunidades Autónomas pueden tipificar infracciones distintas a las enumeradas en la Ley estatal. Con carácter general, son las Comunidades Autónomas las que se encargan de aplicar las disposiciones básicas sobre el régimen sancionador de estas entidades de crédito, si bien la competencia para ello corresponde al Banco de España si la finalidad de las sanciones es proteger la transparencia y la solvencia de las entidades de crédito, así como el cumplimiento de las normas de carácter monetario.

\footnotetext{
${ }^{20}$ Vid., al respecto, el voto particular del magistrado Carles Viver Pi-Sunyer a la sentencia comentada.
} 
La STC 20/1988 señala que las funciones autonómicas relativas a la liquidez y garantía de los depósitos de las Cajas Rurales y cooperativas agrarias y a la canalización de los recursos de las Cajas Rurales son compatibles con la competencia estatal para regular el régimen jurídico de los coeficientes obligatorios, legitimada ésta a partir de la competencia estatal sobre las bases de la ordenación del crédito y sobre las bases y la coordinación de la planificación general de la actividad económica.

Las SSTC 44/1984 y 165/1985, pese a señalar que la dimensión territorial no afecta a las relaciones jurídicas que establezcan las cooperativas con terceros fuera del territorio de la Comunidad, ni a las actividades que realicen con ellos, si tienen carácter instrumental, termina atribuyendo al Estado la autorización para la ampliación de actividades realizadas con terceros no socios que afecten a las realizadas fuera del ámbito comunitario. Igualmente, señala que corresponde al Estado la competencia para calificar la constitución de las cooperativas y la modificación de sus Estatutos.

Similar es la regla aplicada por el Tribunal Constitucional al régimen competencial de las cooperativas de seguros. La STC 86/1989 señala, por una parte, que lo relativo al régimen jurídico de las cooperativas es de competencia exclusiva de ambas Comunidades, de modo que, en relación con estos entes, se da una excepción al régimen competencial de las demás sociedades dedicadas a la actividad aseguradora, determinado por la aplicación de la competencia del Estado sobre las bases de la ordenación de los seguros (art. 149.1.13 CE) y la competencia de las Comunidades Autónomas sobre el desarrollo legislativo y la ejecución sobre los seguros, en el marco de la regulación general del Estado ${ }^{21}$. Sin embargo, al mismo tiempo, son aplicables a la actividad de estos entes las bases de ordenación que el Estado haya establecido pa-

${ }^{21}$ Esta competencia se recoge en los artículos 11.2.a) del Estatuto de Autonomía del País Vasco, 10.1.4 del Estatuto de Autonomía de Cataluña, 15.1.3 del Estatuto de Autonomía de Andalucía, 32.4 del Estatuto de Autonomía de la Comunidad Valenciana y 32.15 del Estatuto de Autonomía de Canarias. Por el contrario, limitan las competencias autonómicas a la función ejecutiva en relación con la materia de crédito, banca y seguros los artículos 39.1.5 del Estatuto de Autonomía de Aragón, 33.12 del Estatuto de Autonomía de Castilla-La Mancha, 28.1.3 del Estatuto de Autonomía de Madrid, 26.15 del Estatuto de Autonomía de Cantabria, 12.14 del Estatuto de Autonomía de Asturias, 11.1.6 del Estatuto de Autonomía de La Rioja, 36.11 del Estatuto de Autonomía de Castilla y León, 12.17 del Estatuto de Autonomía de las Islas Baleares y 9.14 del Estatuto de Autonomía de Extremadura. Como excepción, o más bien como anomalía, ni el Estatuto de Autonomía de Galicia ni el de la Región de Murcia incluyen competencia alguna de dichas Comunidades Autónomas sobre la materia crédito, banca y seguros, más allá de la competencia sobre instituciones de crédito cooperativo, público y territorial y cajas de ahorro. 
ra regular la actividad aseguradora, dentro de los límites de su competencia, a partir de lo cual el Estado puede regular, incluso, y dada la extensión que toman las bases en esta materia, a semejanza de lo que ocurre respecto a la ordenación del crédito, aspectos estructurales de mutuas y cooperativas que son incuestionablemente relevantes para su caracterización e individuación respecto a otras entidades aseguradoras. Se trata de aspectos relativos a la condición de socio, a la posición de los socios respecto a las deudas sociales, a derechos y deberes de los socios, a las características de los órganos de gobierno de la cooperativa, a la inscripción en el Registro Mercantil, etc. Hay que interpretar, en definitiva, que la regulación estatal dimanante de las bases de la ordenación de los seguros se aplica a la ordenación de los derechos y obligaciones de los socios, a la inscripción de las cooperativas y a la estructuración de sus órganos de gobierno en la misma medida que respecto a las demás sociedades dedicadas a la actividad aseguradora, si bien ello permite al Estado regular de modo específico estos aspectos en atención a las peculiaridades de las cooperativas. Lo mismo se afirma respecto a las mutualidades de seguros. Estos pronunciamientos han sido confirmados en sentencias posteriores, como las 35/1992, 330/1994 y 206/1997.

\section{REFLEXIONES FINALES: EL REPARTO DE COMPETENCIAS EN MATERIA DE ASOCIACIONES PASADO POR EL TAMIZ DE LA REALIDAD}

Desde un planteamiento teórico, la doctrina del Tribunal Constitucional acerca de la interpretación de los títulos competenciales autonómicas sobre asociaciones parece permitir un margen amplio para la regulación autonómica de aquellas. Ciertamente, el carácter formalmente exclusivo de las competencias autonómicas sobre determinadas asociaciones sufre la incidencia de la regulación del Estado en esta materia sobre la base del artículo 81.1 de la Constitución y de determinados títulos competenciales que a aquél atribuye la Constitución, entre los que destaca el del artículo 149.1.1 de aquella. Pero, hecha esta salvedad, la STC 173/1998 ha reconocido que la competencia de las Comunidades Autónomas sobre diversos tipos de asociaciones alcanza la facultad de regular de una forma completa y general todos los aspectos de aquellas asociaciones a las cuales extienden su competencia.

Sin embargo, el contenido de la Ley Orgánica 1/2002, mediante la que el Estado ha regulado el derecho de asociación, desmiente en la práctica la amplitud del margen de actuación de las facultades normativas de las Comunidades Autónomas en relación con las asociaciones de su competencia. La Ley 
Orgánica 1/2002 ha regulado los siguientes elementos del régimen normativo de las asociaciones ${ }^{22}$.

a) A partir de su consideración como desarrollo del derecho fundamental de asociación, el objeto y ámbito de aplicación de la Ley, contenido, principios y límites del derecho de asociación, capacidad para constituir asociaciones y formar parte de ellas, prohibición de conceder ayudas por parte de los poderes públicos a las asociaciones que en su proceso de admisión o de funcionamiento apliquen reglas discriminatorias o que promuevan, justifiquen o enaltezcan el odio, la violencia o los delitos de terrorismo, o que realicen actos de descrédito, menosprecio o humillación de las víctimas de los delitos terroristas o de sus familiares, efectos de la inscripción en los Registros de Asociaciones, tanto el nacional como los autonómicos, derecho a formar parte de una asociación y a separarse de la misma, derechos de los asociados, derecho de inscripción, publicidad de los Registros, denegación de la inscripción, tutela judicial, suspensión y disolución judicial de las asociaciones.

b) A partir de su consideración como desarrollo de la competencia del artículo 149.1.1 de la Constitución, relativa a la regulación de las condiciones básicas que garanticen la igualdad en el ejercicio de los derechos y en el cumplimiento de los deberes constitucionales, las condiciones para el ejercicio del derecho de asociación de las entidades públicas, reglas generales sobre fomento, asesoramiento e información a las asociaciones por parte de la Administración, constitución de las asociaciones en lo relativo al acuerdo de constitución, contenido del acta fundacional y de los estatutos, denominación, fijación del domicilio, efectos y práctica de la inscripción registral, normas generales sobre régimen interno y organización de las asociaciones, destino de los beneficios obtenidos por las asociaciones, responsabilidad de las asociaciones, disolución, procedimiento concursal en caso de insolvencia de la asociación, deberes de los asociados, contenido de los asientos del Registro Nacional de Asociaciones, existencia de los Registros Autonómicos de Asociaciones y cooperación y colaboración entre Registros, actos inscribibles y depósito de documentación, régimen jurídico de la inscripción, responsabilidad en casos de cuestaciones y suscripciones públicas y régimen de las asociaciones inscritas con anterioridad a la entrada en vigor de la Ley.

\footnotetext{
${ }^{22}$ Pueden verse en la Disposición Final primera de la Ley los artículos a los que corresponde la regulación de tales elementos.
} 
c) Como ejercicio de la competencia exclusiva del Estado sobre legislación procesal del artículo 149.1.6 de la Constitución, los artículos sobre reparto de competencias entre el orden jurisdiccional contencioso-administrativo y el orden jurisdiccional civil para el conocimiento de los asuntos relativos a asociaciones y sobre inscripción en los Registros de Asociaciones de determinadas comunicaciones de los órganos judiciales.

d) Como aplicación de la competencia de la competencia exclusiva del Estado sobre Hacienda general y Deuda del Estado del artículo 149.1.14 de la Constitución, la regulación de la declaración de asociaciones de utilidad pública y de los derechos, obligaciones, procedimiento para la declaración y otros beneficios de este tipo de asociaciones.

Los demás contenidos de la Ley Orgánica 1/2002, de acuerdo con el apartado quinto de su Disposición Final primera, sólo son de aplicación a las asociaciones de ámbito estatal, lo cual constituye una previsión necesaria habida cuenta de que los Estatutos de Autonomía han ceñido las competencias de las Comunidades Autónomas a las asociaciones que desarrollen principalmente sus funciones en el ámbito de cada Comunidad. Resulta interesante examinar cuáles son estos contenidos aplicables solamente a las asociaciones de ámbito estatal, ya que con ellos el legislador estatal ha expresado también cuál es el concepto que tiene acerca de la extensión de la capacidad de las Comunidades Autónomas para innovar la regulación de determinados elementos de las asociaciones en el marco definido por la Ley Orgánica 1/2002. Podemos entender que el legislador ha considerado que corresponde a las Comunidades Autónomas regular para las asociaciones que desempeñen su actividad principalmente en el ámbito autonómico y que sean de su competencia los elementos que la Ley Orgánica 1/2002 ha entendido que sólo podía regular en relación con las asociaciones de ámbito estatal, a falta de un título competencial específico que apoderara al Estado para la regulación de estos elementos en relación con el conjunto de asociaciones. Estos elementos son la subordinación del otorgamiento de ayudas, subvenciones y beneficios a las asociaciones por parte de los poderes públicos al cumplimiento de los requisitos establecidos para ello, regulación en detalle del régimen interno de las asociaciones a falta de disposición expresa sobre este aspecto en sus Estatutos, régimen de las actividades de las asociaciones salvo lo relativo al destino de sus beneficios, obligaciones documentales y contables, modificación de los Estatutos, liquidación de la asociación, sucesión en la condición de asociado, derechos económicos del socio que decida su separación voluntaria de la asociación, objeto, organización y actividad del Registro Nacional de Asociaciones, 
modo de hacer efectiva la publicidad de los asientos del Registro, medidas de fomento de las asociaciones por parte de las Administraciones públicas y Consejos Sectoriales de Asociaciones.

El principal problema que plantea la Ley Orgánica 1/2002 para la redacción de leyes autonómicas de asociaciones es que ha adoptado una perspectiva material a la hora de regular los elementos de las asociaciones considerados básicos por su conexión con la garantía de la igualdad de todos los ciudadanos en el ejercicio del derecho fundamental de asociación reconocido por la Constitución a partir de la competencia estatal del artículo 149.1.1 de la Constitución ${ }^{23}$. Quiere decirse que la Ley estatal, cuando ha regulado tales elementos básicos, no se ha limitado a hacer una regulación de principios, es decir, no ha llevado a cabo una regulación principialista de las bases, que se limitara a enunciar los principios más básicos del régimen jurídico de las asociaciones dejando un margen de operatividad de las Comunidades Autónomas para complementar dicha regulación, sino que ha pretendido agotar la regulación de tales elementos básicos ${ }^{24}$. Con ello, el margen de actuación de

\footnotetext{
${ }^{23}$ Resulta imposible en este trabajo referirse en detalle a la extensa obra doctrinal que ha tratado sobre esta regla competencial. De hecho, un sector doctrinal ha discutido, incluso, que se trate de una auténtica regla competencial. Puede encontrarse un tratamiento sintético de las principales líneas doctrinales de interpretación de esta norma y de la jurisprudencia del Tribunal Constitucional que se ha ocupado de ella en JIMÉNEZ ASENSIO, R.; ob. cit., págs. 194 y ss., BAYONA ROCAMORA, A.; El derecho a legislar en el Estado autonómico, Tecnos, Escola d'Administració Pública de Catalunya, Madrid, 1992, págs. 140 y ss., y TUDELA ARANDA, J.; Derechos constitucionales y autonomía política, Civitas, IVAP, Madrid, 1994, y «Aproximación a la caracterización jurisprudencial del artículo 149.1.1ํ. de la Constitución», en AA. VV. (coord. S. Martín-Retortillo Baquer); Estudios sobre la Constitución Española en homenaje a Eduardo García de Enterría, t. IV, Civitas, Madrid, 1991, págs. 3.479 y ss.

${ }^{24}$ Vid., sobre las distintas interpretaciones del ámbito de las bases, ALBERTÍ ROVIRA, E.; «La noción de "bases" y el desarrollo estatutario", en AA. VV.; Estudios sobre el Estatuto de Autonomía del País Vasco, t. II, IVAP, Oñati, 1991, págs. 329 y ss., y «Leyes medida y distribución de competencias: un paso más en la interpretación extensiva de las "bases normativas" en la jurisprudencia constitucional. (Comentario a la sentencia del Tribunal Constitucional 179/1985, de 19 de diciembre)», REDC, 18 (1986), 149 y ss., TORNOS I MAS, J.; «Régimen especial de las competencias económicas», en AA. VV.; Comentarios sobre el Estatuto de Autonomía de Cataluña, Institut d'Estudis Autonòmics, Barcelona, 1990-1991, pág. 480, BAYONA ROCAMORA, A.; ob. cit., págs. 172 y ss., JIMÉNEZ CAMPO, J.; "¿Qué es "lo básico”? Legislación compartida en el Estado autonómico», REDC, 27 (1989), 39 y ss., y JIMÉNEZ ASENSIO, R.; ob. cit., págs. 209 y ss. Un amplio trabajo sobre la jurisprudencia del Tribunal Constitucional acerca de la interpretación de los títulos competenciales sobre las bases puede encontrarse en GUTIÉRREZ LLAMAS, A.; La distribución de competencias entre el Estado y las Comunidades Autónomas. Estudio jurisprudencial de la técnica bases más desarrollo, Bosch, Barcelona, 1994.
} 
las Comunidades Autónomas para regular las asociaciones queda, consecuentemente, disminuido. La Ley estatal prefigura la regulación de todos los elementos fundamentales de las asociaciones con un alto nivel de detalle, de manera que a las Comunidades Autónomas, respecto a estos elementos fundamentales, queda, normalmente, poco más que repetir lo ya dispuesto en la regulación del Estado. Ciertamente, los Parlamentos del País Vasco y de Cataluña pudieron disponer de un mayor margen para la creatividad, dado que la Ley 191/1964, que constituía el marco legal estatal vigente en el momento en el que las Leyes de dichas Comunidades Autónomas fueron aprobadas, contenía una regulación más precaria que la de la actual Ley Orgánica 1/2002 y, además, su interpretación requería ser adaptada a las exigencias sustantivas y competenciales derivadas de la Constitución, pero la Ley Orgánica 1/2002, ciertamente, ha configurado un marco bastante preciso que limita en gran medida las opciones reguladoras del legislador autonómico, al menos, por lo que respecta a la regulación de aspectos relativos a los elementos fundamentales de las asociaciones.

El margen para el desarrollo de las funciones legislativas de las Comunidades Autónomas se ve incrementado, lógicamente, en lo relativo a la regulación de los aspectos que la propia Ley Orgánica 1/2002 ha considerado que no pueden ser englobados en el ámbito de alguna competencia específica del Estado. No obstante, ello no es suficiente para difuminar la impresión de que las competencias exclusivas de las Comunidades Autónomas sobre diferentes tipos de asociaciones han quedado convertidas, en la práctica, por una parte, en competencias para la regulación de elementos secundarios de las asociaciones y para la ejecución de la normativa sobre esta materia a escala autonómica y, por otra parte, en competencias con las que las Comunidades Autónomas solamente conservan el control sobre elementos del régimen jurídico de las asociaciones desconectados entre sí, lo que, necesariamente, les tiene que impedir llevar a la práctica una política completa y coherente sobre el hecho asociativo. Queda al lector dar la interpretación que prefiera a este fenómeno, que, por otra parte, es general cuando se trata de la interpretación de las competencias que las Comunidades Autónomas tienen reconocidas en sus Estatutos de Autonomía como exclusivas. Desde una perspectiva puede examinarse como una merma de hecho del ámbito de una competencia exclusiva de las Comunidades Autónomas. Desde la perspectiva contraria puede entenderse como una imprescindible acomodación de la interpretación del ámbito de las competencias de las Comunidades Autónomas a la necesidad de que, por la naturaleza de las cosas y estando en juego la efectividad de un derecho fundamental, la regulación de las asociaciones disponga de un régimen común sólido y extenso en el conjunto del Estado. Examinar ambos argumentos es algo 
que excede del ámbito de este trabajo, en el que solamente pretendíamos poner de relieve la existencia de este fenómeno, tomando como base práctica el caso de la distribución de competencias sobre asociaciones.

Aun saliendo del ámbito propio de las asociaciones, hay que señalar los especiales problemas que presentan, de cara a la determinación del reparto competencial sobre las mismas, las cooperativas, y, en particular, las cooperativas de crédito, por la incidencia en el título competencial autonómico específico sobre cooperativas de aspectos relativos a la legislación mercantil y a las bases de la ordenación del crédito, entre otros que atañen a competencias estatales. Las Comunidades Autónomas tienen reconocida por el Tribunal Constitucional una capacidad amplia para regular las relaciones típicas y singulares que caracterizan a las cooperativas, esto es, aquellas que unen a las cooperativas con sus socios, si bien en el caso de las cooperativas de crédito, la incidencia del título estatal relativo a las bases de la ordenación del crédito ha permitido que el Estado precise con mayor detalle aspectos de la organización de estas cooperativas e, incluso, de las relaciones entre ellas y los socios que las integran, en una línea que ha llegado, incluso, a permitir que el Estado se atribuya facultades de naturaleza ejecutiva en relación con dichas cooperativas. Se trata, nuevamente, de otra muestra de la maleabilidad con la que los títulos competenciales son interpretados por el Tribunal Constitucional en virtud de las circunstancias de la realidad que concurren en ellos, más allá del significado objetivo de los términos en los que se expresan las reglas competenciales que los enuncian. 